\title{
Relación teoría-práctica entre el campo de la formación inicial y el escenario del ejercicio profesional ${ }^{1}$
}

\author{
Theory-Practice Relationship, Between the Initial Training Field and \\ the Professional Practice Scenario ${ }^{2}$
}

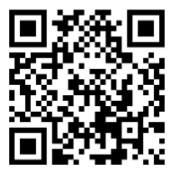

\author{
Gerardo Ignacio Sánchez-Sánchez ${ }^{3}$ \\ Universidad Católica del Maule \\ Talca, Chile \\ correo: gerignsan@gmail.com \\ orcid: http://orcid.org/0000-0003-1694-1406
}

Recibido 17 de junio de 2015 • Corregido 28 de febrero de 2016 • Aceptado 12 de abril de 2016

Resumen. El presente artículo, desarrollado en el campus regional de una universidad, ubicada a 200 millas al sur de Santiago de Chile, se orienta a mostrar cómo 185 estudiantes de pedagogía en educación inicial, básica y media experimentan y representan la relación teoría-práctica a partir de sus inserciones en el medio escolar. El enfoque mixto adoptado se apoya en un cuestionario de opinión y una entrevista semiestructurada administrada en los espacios de tutoría. Los resultados indican que el 39\% de profesionales en formación considera que la teoría y la práctica son realidades opuestas, en tanto el $31 \%$ sostiene que la teoría depende de la práctica. En este escenario, la formación inicial enfrenta el desafío de generar espacios y dispositivos que permitan familiarizar a la persona aprendiz con unos juegos de reglas flexibles y cambiantes para comprender la especificidad del trabajo docente, a partir de una adecuada relación teoría-práctica.

Palabras claves. Relación teoría-práctica, formación inicial, reflexión, tutoría, Chile.

Abstract. This article, developed at the regional campus of a university, located 200 miles south from Santiago de Chile, shows how 185 students of preschool, elementary and middle education, experience and perceive the relationship theory-practice from their insertion in the school environment. The mixed approach adopted relies on an opinion questionnaire and a semi-structured interview administered in the tutoring spaces. The results show that $39 \%$ of the teaching students believe that theory and practice are opposite realities, while $31 \%$ say that the theory depends on practice. In that scenario, the initial teaching training faces the challenge to create spaces and devices that allow the teaching student to get familiar with a few sets of flexible and changing rules to understand the specificity of the teaching process, from a proper theory-practice relationship.

Keywords. Theory-practice relationship, initial teaching training, reflection, tutoring, Chile.

\footnotetext{
${ }^{1}$ Proyecto de investigación financiado por el Plan de Mejoramiento UCM1310 de Formación Inicial de Profesores.

${ }^{2}$ Research project funded by the UCM1310 Improvement Plan Initial Teacher Training.

${ }^{3}$ Doctor en Ciencias de la Educación, Magister en Política y Gestión Educacional, Profesor de Historia y Geografía. Académico de la Facultad de Ciencias de la Educación de la Universidad Católica del Maule. Vasta experiencia en procesos de formación práctica y evaluación del aprendizaje en escuelas y liceos del sistema escolar municipal de la región del Maule, Chile. Ha publicado el texto Aprender a enseñar situando las tareas del profesor. Ha dirigido múltiples investigaciones y expuesto en varios congresos nacionales.
} 
doi: http://dx.doi.org/10.15359/ree.20-2.17

URL: http://www.una.ac.cr/educare

CORREO: educare@una.cr

\section{Introducción}

En la actualidad, existen intensos cuestionamientos planteados a la formación inicial docente, en términos de no proporcionar las herramientas necesarias que permitan hacer frente a las demandas derivadas de los contextos escolares, y de las transformaciones socioculturales. Estas interrogantes terminan por instalar discursos que, situados desde el deber ser o desde aproximaciones normativas e ideales, escasamente contribuyen a develar la complejidad que implica la formación y el trabajo docente para quien lo ejerce, haciendo distante su sintonía con los requerimientos del aula.

Tengamos presente que el profesor o profesora es, "ante todo, un profesional de la articulación del proceso de enseñanza-aprendizaje en situación; un profesional de la interacción de las significaciones compartidas"(Altet, 2010, p. 38), lo cual pone de manifiesto que la profesión docente requiere determinada especificidad a la cual la formación inicial tendría que apostar.

Es importante señalar que diseñar una formación para profesorado supone analizar todas las dimensiones involucradas en el ejercicio del rol y, a partir de ello, decidir las cuestiones a ser cubiertas en la formación, si lo que se pretende es propiciar su desarrollo.

No siempre las propuestas formativas se condicen con la diversidad de roles asociados a la docencia.

Desde una tradición tecnocrática se "establece una clara distinción entre conocimiento teórico y conocimiento práctico, y entiende el segundo como una aplicación del primero" (Diker y Terigi, 1997, p. 115), en tanto la tradición hermenéutica-reflexiva "parte del supuesto que la enseñanza es una actividad compleja que se desarrolla en escenarios singulares, claramente determinada por el contexto, con resultados siempre en gran parte imprevisibles, y cargada de conflictos de valor" (Diker y Terigi, 1997, pp.116-117). Por consiguiente, estos modelos de formación están sustentados por otras distintas concepciones de articulación teoría-práctica.

Desde la perspectiva técnica, se propicia una parcelación de las habilidades que el profesorado debe desarrollar para movilizarse en la acción, a partir de una concepción sumativa de la adquisición de conocimientos; en tanto desde una mirada reflexiva, el sujeto profesional es autónomo y un analista de situaciones de carácter singular, capaz de tomar sus decisiones (Paquay y Wagner, 2005).

Es importante rescatar estos modelos por sus supuestos sobre la práctica docente y porque, de alguna manera, se encuentran más o menos institucionalizados, incorporados a las prácticas y a las imágenes que disponemos para pensar el trabajo y la formación docente.

En un escenario de preocupación por la formación inicial docente, resulta comprensible la atención prestada al tipo de conocimiento que articula el trayecto formativo; los contextos donde dicho proceso se hace efectivo y los actores que lo protagonizan. A partir de estos tres ejes, las propuestas de formación inicial han asumido, a lo largo del tiempo, distintas racionalidades. 
En relación con el tipo de conocimiento "se transita de una tendencia donde el conocimiento teórico disciplinar se constituye en eje predominante en los programas de formación, a una donde el conocimiento práctico y su desempeño se configura como eje central en los programas de formación" (Fuentealba y Sánchez, 2013, p. 66).

En cuanto a los contextos, la formación se ha ido ampliando desde los espacios universitarios a los contextos de aula escolar; y en el caso de los actores, desde la consideración monopólica del personal académico universitario, a la valoración del profesorado guía del establecimiento escolar y al mismo estudiantado de pedagogía, que con sus representaciones e imágenes preformativas también juega un papel crucial (Fuentealba y Sánchez, 2013).

La problemática actual sigue siendo la presencia de:

una lógica dicotómica donde cada uno de los componentes mencionados terminan siendo leídos desde la preponderancia de uno sobre otro, es decir, se mantiene la tensión entre conocimiento teórico $\mathrm{v} / \mathrm{s}$ conocimiento práctico; espacio universitario $\mathrm{v} / \mathrm{s}$ espacio escolar como medio de aprendizaje y académico $\mathrm{v} / \mathrm{s}$ [profesor] del medio escolar como agente formativo, en suma lo que Schön $(1987,1998)$ llamó como racionalidad técnica, o lo que Day (1999) denominó concepción aditiva, o lo que Russell y Korthagen (1995) Ilaman modelo de aplicación de la teoría. (Fuentealba y Sánchez, 2013, p. 67)

En la base de la discusión en torno a una adecuada formación docente se encuentran las relaciones siempre complejas entre la teoría y la práctica. Y el desafío que emerge está en lograr equilibrar una práctica no reductible a un conjunto de dispositivos técnicos, pero tampoco presa de la sorpresa permanente.

Lo anterior constituye el asunto central, tanto en la tarea docente como en su formación, pues se trata de precisar hasta qué punto la actuación del profesorado está informada por conocimiento teórico, pero regida por esquemas que rebasan por completo aquel conocimiento. Se insiste "en que la transformación de conocimiento previsto por la teoría en conocimiento útil para la acción no es un proceso de aplicación sino de reconstrucción situacional" (Diker y Terigi, 1997, p. 118). Por tanto, el problema formativo es qué tipo de balance se establece entre teoría y práctica en la formación, vista su compleja relación en la tarea docente. La respuesta a ello viene condicionadas por los modelos formativos adoptados. De acuerdo con Carr y Kemmis (1990) citado por Diker y Terigi (1997):

diremos que toda teoría demuestra su capacidad al explorar de un modo sistemático y riguroso una serie de problemas; pero, en el caso de la teoría educativa, estos problemas tienen su fuente en la práctica (pp. 119-120). 
doi: http://dx.doi.org/10.15359/ree.20-2.17

URL: http://www.una.ac.cr/educare

CORREO: educare@una.cr

De acuerdo con Terigi (1995), citado en Diker y Terigi, (1997) de lo que se trataría es de lograr modos de organizar adecuadamente el trayecto formativo con una racionalidad que evite caer en "la tendencia aplicacionista (la práctica como aplicación de los fundamentos teóricos) o en la tendencia ejemplificadora (la teoría como explicación posterior de la práctica) (p. 122).

Es preciso reconocer que "el docente no abandona la teoría, muy contrariamente a eso: primeramente, la cuestiona a la luz de los indicios que posee y, más tarde, la vuelve a cuestionar según los resultados de su intervención" (Fuentes, Vásquez y Vergara, 2013, p. 41).

En consecuencia, enfrentado a la tarea de formar el profesorado, los procesos de la práctica están llamados a ocupar un espacio central

Sin embargo, no es del todo claro cómo, para qué y bajo qué circunstancias es posible lograr que dicho espacio, el de la formación en terreno como primera experiencia de inserción, se constituya en un espacio que favorezca no sólo un buen desempeño profesional sino que al mismo tiempo permita ir construyendo los cimientos de un ejercicio profesional que se nutra tanto de lo que sucede al interior de la universidad como de lo que sucede en el mundo escolar. (Correa, Cividini y Fuentealba, 2013, p. 9)

La práctica tiene como objetivo "permitir la aproximación gradual de los estudiantes al trabajo profesional, y facilitar el proceso por el cual los futuros profesores construyen conocimiento pedagógico, desarrollan en forma personal teoría y práctica de la enseñanza y el aprendizaje y, sobre todo, aprenden a enseñar" (Avalos, 2002, p. 113).

Teniendo presente que no se puede enseñar todo en la formación inicial a un futuro profesor o profesora, queda claro que tampoco se puede actuar sin un marco conceptual -epistemológico y pedagógico-, que ayude a potenciar la reflexión que debe realizar el profesorado en formación en el desarrollo de sus aprendizajes acerca de la enseñanza de su disciplina Esto nos plantea un estilo de trabajo conjunto, entre un formador y aprendiz de la enseñanza (Padilla y Pedreros, 2013, p. 71), para la comprensión y ejercicio del trabajo docente, teniendo en consideración que se trata de un grupo humano que posee "diversidad y heterogeneidad de ideas y experiencias al momento de resignificar epistemológica y didácticamente el 'conocimiento a enseñar'"' (Montero, 2001; Puig y Calderón, 1996 y Zeichner, 1990, citados en Padila y Pedreros, 2013, p. 71).

El aprendizaje de un oficio complejo como la enseñanza debe abarcar una teoría asociada a la práctica y una práctica que derive de esta teoría. Vincular una y otra implica... que cada formador, en su propio contexto, suscite el planteamiento de los aspectos teóricos articulándolos con las problemáticas que vive el estudiante. (Bélair, 2010, p. 105) 
En el logro de tal aprendizaje y reconociendo su carácter procesual, el estudiantado en práctica, enfrentado al territorio del aula, a partir de ciertos proyectores conceptuales que lo dotan de una percepción parcial de la realidad que condiciona su dominio de las situaciones, constata que nada o muy poco se comporta como estaba previsto, y se ve necesitado de reglas flexibles y cambiantes, pues la explicación teórica omnisciente no le sirve, pues resulta parcial. "Una formación profesional que gire, en un principio, en torno a la comprensión y al análisis teóricos de la intervención contribuye a retrasar la aprehensión de la economía pedagógica por el hecho de que incita a realizar una lectura fragmentaria de las situaciones" (Carbonneau y Hétu 2010, p. 128).

\section{Metodología}

Este artículo informa parte de la investigación Visión del trabajo docente desarrollada por estudiantes en procesos de inserción al aula (Sánchez, 2014-2015), desarrollada en el contexto de la Facultad de Ciencias de la Educación. El objetivo principal de dicha investigación fue mostrar cómo el estudiantado de pedagogía en educación preescolar, básica y media, experimenta y representa la relación teoría-práctica.

Como parte del proceso de inserción al espacio escolar, el estudiantado de pedagogía asiste a sesiones periódicas detutoríaen la universidad, defrecuencia semanaly con una duración aproximada de sesenta minutos en modalidad individual o grupal, según las necesidades emergentes.

Se trabajó con una muestra intencionada de 185 estudiantes de ambos sexos, que enfrentan procesos de práctica.

Los criterios para elegirlos fueron:

- Estar cursando el último semestre de las titulaciones de preescolar, básica y media.

- Aceptaran participar de forma voluntaria y anónima.

La población que participó en el estudio se distribuyó de la siguiente manera (Tabla 1):

Tabla 1

Distribución de estudiantes participantes del estudio

\begin{tabular}{lcc}
\hline Titulación & $\mathrm{F}$ & $\%$ \\
\hline Educación Preescolar & 31 & 16.7 \\
Pedagogía Básica & 40 & 21.6 \\
Pedagogía Media & 114 & 61.6 \\
\hline Total & 185 & 99.9 \\
\hline
\end{tabular}

Nota: Elaboración propia. 
doi: http://dx.doi.org/10.15359/ree.20-2.17

URL: http://www.una.ac.cr/educare

CORREO: educare@una.cr

El diseño utilizado fue de tipo transeccional no experimental con un nivel de profundidad exploratorio, utilizando metodología cuantitativa y cualitativa, a través del método de estudio de casos múltiples (Hernández, Fernández y Baptista, 2010).

El abordaje metodológico requirió, en un primer momento, de una aproximación de carácter extensivo (de tipo cuantitativo) a través de la administración de un cuestionario dirigido a la población en estudio. Este permitió obtener antecedentes para configurar tendencias generales respecto de la relación teoría-práctica. Para su administración, se realizó una convocatoria ampliada al estudiantado en práctica profesional a través de las personas coordinadoras de práctica de las distintas titulaciones, quienes gestionaron la aplicación del instrumento.

En un segundo momento, en una aproximación de carácter intensivo se seleccionaron casos que permitieran profundizar en aquellos aspectos más significativos aparecidos en el primer momento, de la forma como se representan y significan los vínculos entre teoría y práctica. El conjunto de elementos mencionados anteriormente dio cabida a entrevistas semiestructuradas administradas a 30 estudiantes de las distintas titulaciones. Los sujetos entrevistados fueron seleccionados de acuerdo con los siguientes criterios:

- Casos seleccionados según intensidad con la que los rasgos, los procesos, las experiencias se producen.

- Casos particularmente típicos de cada una de las tendencias evidenciadas en el estudio (Flick, 2007).

Desde el punto de vista cualitativo, la metodología se propuso describir y comprender las realidades subjetivas desde las percepciones de los actores sociales que competen a este estudio, es decir, estudiantes de educación parvularia, pedagogía básica y enseñanza media. La metodología cualitativa es la adecuada para explorar este constructo de la relación teoría-práctica, ya que es un procedimiento que permite reconstruir conceptos, analizando las diferentes relaciones que surjan entre ellos, a partir del relato de los actores (Krause, 1995).

Finalmente, cabe mencionar que se decidió presentar los datos como un todo, sin contextualizar sistemáticamente según las distintas titulaciones o carreras. Se trató de identificar aquí no lo que distingue las diferentes titulaciones, sino la experiencia común de los profesionales en formación que enfrentan su práctica profesional en los diversos contextos de la región y que son acompañados en el proceso, por personal tutor que la institución formadora dispone. 


\section{Resultados}

\section{Respecto a la relación teoría-práctica (tabla 2)}

Tabla 2

Relación teoría-práctica

\begin{tabular}{llc}
\hline Afirmación & F & $\%$ \\
\hline "La universidad solo nos enseña lo que dicen los libros; la práctica de la escuela es otra cosa". & 73 & 39.4 \\
"Debemos enseñar desde lo concreto, pues la psicología genética indica que no hay & 34 & 18.3 \\
$\begin{array}{l}\text { abstracción sin antes existir experiencias concretas". } \\
\text { "No existen teorías generales en educación. Solo podemos construir explicaciones }\end{array}$ & 58 & 31.3 \\
$\begin{array}{l}\text { específicas a partir de la realidad donde trabajamos". } \\
\text { "Teoría y práctica una relación de interdependencia". }\end{array}$ & 20 & 10.8 \\
\hline Total & 185 & 99.8 \\
\hline
\end{tabular}

Nota: Elaboración propia.

Es posible constatar que para el $39.4 \%$ de docentes en formación que enfrentan el proceso de práctica, la relación entre esta última y la teoría se plantea en la lógica de "Teoría y práctica son realidades opuestas; la primera es universal, la segunda es particular y depende de los contextos"; razón por la cual según su criterio "la universidad sólo nos enseña lo que dicen los libros; la práctica de la escuela es otra cosa"

Por otro lado, para el 31.3\%, la relación se concibe en términos de "la teoría depende de la práctica. Es necesario saber cómo se hacen las cosas para luego entender qué son las cosas"; razón por la cual "No existen teorías generales en educación. Solo podemos construir explicaciones específicas a partir de la realidad donde trabajamos"

Desde otra perspectiva, para el $18.3 \%$ de profesionales en formación "la práctica depende de la teoría. Toda práctica posee una teoría que orienta la acción"; lo cual supone que "Debemos enseñar desde lo concreto, pues la sicología genética indica que no hay abstracción sin antes existir experiencias concretas"

Finalmente, Ilama la atención el 10.8\% de los estudiantes que optan por “Teoría y práctica una relación de interdependencia". Para ellos, una adecuada formación implica una estrecha relación teoría y práctica.

Ahora bien, cuando se profundiza en los relatos generados por profesionales en formación respecto a estas formas de entender la relación teoría-práctica, como parte de las entrevistas realizadas, emergen matices interesantes de considerar. 
doi: http://dx.doi.org/10.15359/ree.20-2.17

URL: http://www.una.ac.cr/educare

CORREO: educare@una.cr

En relación con:

\section{La universidad solo nos enseña lo que dicen los libros; la práctica de la escuela es otra cosa ó primero se estudia y aprende y luego se practica}

Se observa la presencia de estudiantes que no logran establecer relaciones de interacción entre las dimensiones de la teoría y la práctica. Para tales estudiantes, existiría una institución formadora que entrega elementos de carácter declarativo o conceptual que poco o nada se relacionan con las características y necesidades de la práctica. Esta práctica que para el grupo representa la realidad y el espacio del ejercicio efectivo de la docencia, tendría otra textura y naturaleza que la teoría no logra reflejar. Así se explican estos relatos cuando señalan:

A mi modo de ver las cosas, la universidad nos entrega herramientas cognoscitivas para que las aprendamos (talleres, guías, mecanismos, literaturas, entre otros) y posteriormente llevarlos a la práctica (realidad) y poder entregarlos de la manera más ideal posible, ahora bien, esa pretensión se difumina en la realidad, en los establecimientos educacionales y con los alumnos que enfrentamos. (C2)

Aprender se aprende en la práctica, muchas veces lo que entrega la universidad son cuestiones ideales, teóricas, distanciadas de la realidad. (F15)

Muchas ocasiones nos encontramos con situaciones que se escapan a los conceptos teóricos que se nos enseña en la educación superior, recurriendo a criterios, recursos personales $e$ intuitivos o competencias desarrolladas a través del tiempo. $(\mathrm{H} 3)$

Cuando estoy en la sala de clase y enfrento a mis estudiantes, lo hago apelando a mi sentido común o a mis recuerdos de profesores. (H7)

Mi propuesta se sostiene en que las teorías en la educación sirven como antecedentes que orientan el trabajo del profesor, pero la realidad de verse enfrentado a impartir clases, supera cualquier teoría relacionada ya que lo que ocurre en un salón de clases, difícilmente se acerca a la visión parcelada o que describe una teoría o estudio. (12)

Puede que nos enseñen los contenidos básicos o nos traten de decir "cómo es el mundo laboral mostrarnos libros de clases, videos de clase, que observemos clases, pero ser profesor es otra cosa totalmente distinta, e incluso opuestas. En los libros todo se ve color de rosa (lindo) en la práctica se ve la verdad... (18) 
En la Universidad normalmente se nos prepara para poder ser capaces de transmitir lo que en algún momento un profesor nos dijo, trabajamos a través de estímulos y raramente por intuición, el trabajar en la Escuela de Hoy, es muy distinta a como los libros describían ésta, debido a que la realidad en que se encuentra un joven de hoy, sin identidad propia, genera dificultad en el sistema escolar. Me baso en lo que veo día a día en mi Colegio, donde los alumnos no tienen interés de estudiar y el rol del profesor es fundamental para su avance en la vida, ya que si no se aferran a esa posibilidad, serán futuros desertores de la tan ansiada educación (I9)

En muchas ocasiones nos encontramos con situaciones que se escapan a los conceptos teóricos que se nos enseña en la educación superior, recurriendo a criterios, recursos personales e intuitivos o competencias desarrolladas a través del tiempo. $(\mathrm{H} 3)$

Los relatos permiten advertir un claro cuestionamiento a la institución universitaria en relación con el contenido de la formación y el impacto que efectivamente logra, dado que los requerimientos de la práctica lo desbordan y superan, llevando a sus practicantes a movilizar recursos personales, intuición o experiencias preformativas. La práctica emerge, entonces, como el espacio que les permite aprender y ser docente.

Asimismo, el contenido de la formación tiende a quedar anclado a declaraciones normativas y prescriptivas que proyectan una imagen ideal de la docencia, en tanto, la realidad, reflejada en la práctica de lo que sucede en el aula, se manifiesta en situaciones inciertas y complejas, que explican el uso de recursos de carácter personal para desenvolverse. Reconocen que el perfil del estudiantado y el dinamismo de los contextos de inserción, tienden a explicar esta mirada respecto a la relación teoría-práctica.

\section{Debemos enseñar desde lo concreto, pues la psicología genética indica que no hay abstracción sin antes existir experiencias concretas}

Una concepción tradicional de la formación otorga un lugar central al conocimiento, pues para convertirse en docente habría que conocer -antes de aplicar- las bases teóricas de la disciplina que se enseña

Para los grupos de profesionales en formación, primero se estudia y aprende lo necesario, las herramientas como refieren, para luego transferirlo o aplicarlo. Emerge la creencia según la cual la formación sigue una progresión preestablecida, basada en una concepción "sumativa" de la adquisición de conocimientos, que se estructuran como si los procedimientos profesionales pudieran ser construidos paso a paso, uno tras otro. También surgen nociones planteadas desde el deber ser. En sus relatos indican que: 
doi: http://dx.doi.org/10.15359/ree.20-2.17

URL: http://www.una.ac.cr/educare

CORREO: educare@una.cr

El constructivismo se enmarca en que los niños asuman un rol activo-participativo, para ello, es indispensable el hacer, aprender haciendo, a través de experiencias concretas que lo Ileven a la abstracción de conceptos Esto facilita en el estudiante un aprendizaje que le dejará conocimiento permanente. Para mí así se opera en el espacio de aula. (B4)

Es fundamental tener los conocimientos teóricos para poder aplicarlos en la sala de clases. Si no tengo claro que tengo que enseñar voy a tener problemas en el aula. (C5)

Creo que esta concepción es la que debiera identificar al estudiante en su práctica profesional, el quehacer docente se ve plasmado en la práctica y el alumno en su internado ve como sus conocimientos teóricos, se hacen aprendizaje en lo práctico. (F39)

Tener la oportunidad de ir a prácticas intermedias no sólo le sirve para conocer el mundo en el que trabajará sino también aplicar lo que me están enseñando. Necesita ir poniendo en práctica los conocimientos aprendidos en su carrera profesional. (P2)

Es importante el proceso de prácticas progresivas, para que las alumnas sitúen y den sentido a los conocimientos del aula universitaria, a través del análisis y la reflexión que produce la tensión teoría-práctica. ( $\mathrm{P} 3)$

La teoría es imprescindible, para que luego en la práctica del aula nos forjemos como profesor. Las distintas realidades educativas, particulares, subvencionadas, municipales, etc., deberían conocerlas todas y estar preparados para solucionar problemas emergentes en toda clase de realidades. (C1)

Concuerdo con esta opción, por cuanto la teoría es esencial en el proceso educativo, para lograr entender la realidad del aula y actuar sobre ella. El sustento teórico nos asegurar las explicaciones para abordar nuestra práctica. $(\mathrm{H} 5)$

Creo que ésta es la que más se acerca a mis creencias. Significa por cierto un dominio mayor de las teorías educativas y de los modelos pedagógicos que las sostienen, de modo de tener las herramientas que les permitirían tomar buenas decisiones dentro del contexto "aula". (H6)

Existen siempre dos pasos previos, antes de la aplicación y que están vinculados al conocer y comprender; para luego aplicar. Un ejemplo simple: "Enseñanza de una adivinanza para un niño de 2 años 5 meses" 
doi: http://dx.doi.org/10.15359/ree.20-2.17

URL: http://www.una.ac.cr/educare

CORREO: educare@una.cr

Para llevar a cabo esta tarea la estudiante debe conocer cuál es la metodología más adecuada para enseñar una adivinanza a un niño de medio menor de acuerdo a sus características, (tiempo de concentración, formas para captar más su atención) una vez que conoce aquello selecciona la adivinanza el método que utilizará para enseñarla. Estando listo esto, se efectúa el ejercicio práctico. (P5)

Es importante asegurar que nuestras acciones no sean improvisadas, no sean ensayos y error; puesto que al estar trabajando con seres humanos en una edad de tanta sensibilidad como es el nivel de educación parvularia marcan diferencia desde lo positivo o negativo nuestras intervenciones. Por lo tanto, son claves las oportunidades teóricas para intervenir en la realidad educativa. (P4)

Aun cuando por la experiencia personal donde más se aprende es en la práctica, no se puede concebir una práctica sin los conocimientos y fundamentos teóricos que brinda la universidad, para realizarla. (M1)

La comprensión cabal de un nuevo conocimiento se basa en uno anterior, es decir la construcción del conocimiento se sustenta en otro, por lo tanto primero es la teoría y desde ella la práctica. (M2)

La teoría entregada en los diferentes módulos debería asegurar las herramientas para enfrentar los diferentes contextos socioculturales a que se verán enfrentados los futuros docentes en su práctica. (F2)

Este grupo de estudiantes de pedagogía, cree posible enfrentar la complejidad que reconocen en los contextos escolares, a partir de conocimientos y procedimientos que deben ser adquiridos en forma previa, como parte de la teoría.

Obviamente, es importante la experiencia previa para algunos eventos, sin embargo, la teoría debe preceder a la práctica o darse paralelamente en aspectos tan relevantes como la educación, donde se trabaja con la integridad individual y social. (C3)

Personalmente creo que un buen profesional debe primero adquirir el conocimiento necesario para luego ponerlo en práctica y no cometer errores irreparables, más aún, perfeccionarse día a día. (F1)

Esfundamentalqueel alumnoantes deenfrentarsupráctica, demuestrequetienelas herramientas (conocimientos) necesarios para desempeñar la labor docente, el alumno en práctica no puede "practicar" de la nada, necesita conocimientos sólidos para poder llevarlos a la práctica. (14) 
doi: http://dx.doi.org/10.15359/ree.20-2.17

URL: http://www.una.ac.cr/educare

CORREO: educare@una.cr

El alumno debe tener una base sólida en conocimiento relativo a su especialidad para luego lograr utilizar las mejores estrategias para que se produzca un aprendizaje significativo en los alumnos. (15)

Para entregar -enseñar- conocimientos, y posteriormente, aplicarlos primero el estudiante debiese haberlos incorporado e internalizado el mismo. De este modo, se les facilitará la entrega-la transmisión-de sus propios conocimientos a sus alumnos. (17)

Se advierte la presencia de una mirada aún incompleta de la tarea docente, con visiones más bien estereotipadas de la relación teoría-práctica, concebida en términos de orden y dependencia de una respecto a la otra, y otorgando prioridad a la teoría, la cual emerge como el soporte para el enfrentamiento de la realidad y con capacidad de respuesta. Emerge en el planteamiento de este grupo de estudiantes de pedagogía una racionalidad empírico-analítico (Boggino y Rosekrans, 2004), al momento de comprender la relación teoría-práctica.

La teoría se valora en tanto les ha de permitir entender la realidad del aula y actuar sobre ella, apelando a la necesidad de un dominio mayor de las teorías educativas y de los modelos pedagógicos que las sostienen, de modo que obtener las herramientas que les permitirían tomar buenas decisiones dentro del contexto "aula" es fundamental.

Frente a una realidad educativa -el mundo de los colegios- que visualizan como compleja, pretenden su afrontamiento desde lógicas que tienden a la separación y al reduccionismo de la teoría, sin entender las intersecciones complejas teoría-práctica. Probablemente la razón radica en el imperativo de asegurar que las acciones en el aula no sean improvisadas ni caigan en la dinámica de ensayo y el error, aspectos que creen pueden controlar a partir de las oportunidades y herramientas teóricas que, en forma previa, esperan de la institución formadora.

\section{No existen teorías generales en educación. Solo podemos construir explicaciones específicas a partir de la realidad donde trabajamos}

En esta lógica, la práctica permite a los grupos profesionales en formación adquirir esquemas de acción, es esta donde más se aprende, pues se enfrentan a saberes prácticos intensamente contextualizados. En este contexto emergen los siguientes relatos:

Es finalmente en la práctica donde el alumno logra modelar su perfil específico de docente como una mezcla de conocimiento teórico, personalidad y formación inicial y las necesidades que el propio contexto del establecimiento educativo le presenta como desafío profesional. (B1) 
No podemos aplicar por igual una misma planificación a realidades muy disímiles; cuando la realidad, las expectativas de los padres y los intereses de los estudiantes van por caminos muy opuestos. Deberíamos adecuar el aprendizaje al entorno, a la realidad concreta. Tal vez así lograríamos una educación más adecuada a las necesidades de la sociedad actual. (C7)

No desconozco las teorías existentes, creo que es muy útil conocerlas, estudiarlas y aplicarlas, pero considero que el momento en que nos enfrentamos a los estudiantes, a sus problemáticas, a sus dudas, a su mundo; descubrimos realmente si seremos capaces de colaborar en su crecimiento como persona, donde los contenidos son el medio para acercarnos y avanzar también en su aprendizaje académico. (C8)

Reconocer la individualidad, ambiente, historicidad, elementos demográficos, etc. son elementos decisivos quese deben considerar antes de implementar una unidad de aprendizaje, y que uno los descubre en el terreno y a partir de estas necesidades emerge la preocupación por la teoría. (F5)

Creo que a pesar de tener muchos conocimientos en educación no podemos regirnos por normas o teorías generales ya que cada Escuela o lugar de trabajo tiene un entorno, una realidad completamente diferente y las personas con las que nos relacionamos pertenecen a ella con toda su historicidad tomando en cuenta sus diferentes actividades y valorándolas en su desempeño. (F6)

Enfrentado a la diversidad de contextos, de lo que se trata es de hacer lo mejor dentro de lo posible, para desarrollar en los estudiantes competencias mínimas para su desenvolvimiento social. Quizás esto no concuerde con las declaraciones de las autoridades y directivos, pero suele ser la dramática y penosa realidad. (H7)

En relación con esta mirada, el marco sociocultural, el contexto de aula, las características estudiantiles configuran un trabajo docente singular y abierto a la incertidumbre con una complejidad que la teoría no logra capturar, razón por la cual la "práctica" determina la teoría. Desde su perspectiva, la práctica es el momento en que se enfrentan a sus estudiantes, a sus problemáticas, a sus dudas, a su mundo; en que se descubre realmente la labor, y la posibilidad de ser o no capaz de colaborar en su crecimiento como persona. En este sentido, la teoría omnisciente no tiene capacidad de respuesta. Por último, se reconoce el carácter multiforme de la práctica, desde el cual emerge su potencial formador. 
doi: http://dx.doi.org/10.15359/ree.20-2.17

URL: http://www.una.ac.cr/educare

CORREO: educare@una.cr

\section{Teoría y práctica una relación de interdependencia}

En una visión más compleja de la relación teoría-práctica están aquellos grupos de estudiantes que vislumbran las relaciones de interconexión que es preciso reconocer entre ellas para comprender la especificidad del trabajo del profesorado. Nos encontramos con los siguientes relatos:

Si yo no puedo aprender del modo que tú enseñas, ¿me podrías enseñar de modo que yo aprenda?, es lo que he reflexionado en mi formación. La realidad en la cual me inserté ha hecho cuestionarme constantemente los aprendizajes que están logrando mis alumnos, el alto número de alumnos de integración y vulnerabilidad que llegan al Liceo me están obligando a tratar de hacer mío el lema anterior y alcanzar diariamente lo que espero de ellos. (H8)

Observo, reflexiono, construyo. Me parece que lo principal es que como alumno sepa reconocer las capacidades que he desarrollado a través de mi proceso de formación y que a partir de ellas trate de abordar el proceso de practica con compromiso y responsabilidad, con una mirada de aprendiz, que reflexione y reconstruya a partir de la teoría y la realidad en la cual está inserto. (M4)

La gran fortaleza de estos periodos de aproximación o inserción al proceso educativo es que como estudiantes podemos ir relacionando los conocimientos teóricos adquiridos en nuestros estudios formales con las experiencias prácticas que vivimos en las instituciones donde nos insertamos. Tenemos que entender las interacciones que se producen entre teoría y práctica. (I1)

En Educación, es importante el experimento con la teoría y el manejar varias teorías sobre distintas estrategias de enseñanza, aprendizaje, evaluaciones, etc .Mientras más teorías conozca más herramientas tendrá el profesor para dar respuesta a los múltiples problemas que se presentan en la sala. (B4)

Necesitamos Profesionales de la Educación que sean capaces de reflexionar continuamente en torno a sus prácticas pedagógicas y el entorno en el cual se desarrollan, para entregar un trabajo serio a estudiantes que deben ser educados no sólo para el mundo del trabajo sino para la vida. Por lo mismo, sabemos que tanta teorización alejada de la práctica vivencial no sirve de mucho. (C9)

Creo que no existen generalidades ni recetas mágicas para tener éxito en el desempeño de la docencia, cada realidad es única, cada colegio en un mundo único y eso requiere reflexión para convertirse en un profesor. (16)

Para mí son un mismo proceso cuya denominación de "teoría" es bajo mi óptica, la entrega y procesamiento por parte de la estudiante de la información acumulada y entregada por otros, respecto de lo que se espera saber. Este conocimiento proviene de teóricos, tutores, pares, 
familia, sociedad, otros profesionales y mi propio saber preconcebido. Pero si bien es cierto la teoría gana prelación y me parece un orden lógico, creo que esta debe ser entregada primero pero por escaso tiempo, para luego la práctica darle alcance y amalgamarse en un proceso en que una no se distinga de la otra sino formen un concepto mixto, que hasta ahora a falta de otro mejor se denomina teórico-práctico. (P1)

En general todas las teorías tienen su origen en una práctica, en nuestro caso la teoría relata lo que pasa en la sala de clases, lo ordena, lo interpreta y lo lleva al estudio detallado para aprender mejor de ellas, por esto la reflexión es la convergencia de ambas. Por un lado es tan importante la experiencia en terreno, y de ahí la reflexión de lo que se vivió, refuerza esto la teoría cognitiva de Piaget nos dice que las Operaciones Formales, donde el hombre adulto, o adolescente logra la abstracción sobre conocimientos concretos observados que le permiten emplear el razonamiento lógico inductivo y deductivo. Por tanto no hay teoría sin práctica. La práctica educativa va más allá del cómo se hacen las cosas, una buena práctica siempre es teoría, ya que a través de ella podemos mejorar por el relato y la reflexión. $(\mathrm{H} 1)$

Todos estos relatos plantean una relación dinámica y necesaria teoría-práctica, mediada por la capacidad de reflexión del profesorado en formación, que enfrentado a un trabajo de carácter situado, debe aprender a comprender para perseverar en él. Ello demanda un dispositivo formativo donde se hace conveniente que el alumnado de pedagogía se relacione con las unidades educativas desde el primer año de su carrera para que aprecie la realidad en la cual va a trabajar y no sufra el duro golpe con la realidad en el último año. Lo anterior, pues la teoría y la práctica están íntimamente ligadas. La práctica sirve para entender y confirmar la teoría. La teoría sirve para evitar improvisaciones y dirigirla en forma ordenada.

\section{Respecto al funcionamiento de la tutoría en procesos de práctica profesional}

\section{Tipo de establecimiento donde realizan práctica (tabla 3)}

Tabla 3

Tipo de establecimiento donde realizan práctica

\begin{tabular}{lrr}
\hline Tipo de establecimiento & $\mathrm{f}$ & \multicolumn{1}{c}{$\%$} \\
\hline Municipal-público & 75 & 40.5 \\
Particular subvencionado & 81 & 43.7 \\
Particular pagado & 18 & 9.7 \\
Fundación Integra & 4 & 2.1 \\
Junta Nacional de Jardines infantiles (JNJ) & 7 & 3.7 \\
\hline Total & 185 & 99.7 \\
\hline
\end{tabular}

Nota: Elaboración propia. 
doi: http://dx.doi.org/10.15359/ree.20-2.17

URL: http://www.una.ac.cr/educare

CORREO: educare@una.cr

\section{Ubicación del centro de práctica (tabla 4)}

Tabla 4

Ubicación centro de práctica

\begin{tabular}{lrr}
\hline Ubicación del centro & $\mathrm{f}$ & \multicolumn{1}{c}{$\%$} \\
\hline Talca & 143 & 77.2 \\
Linares & 20 & 10.8 \\
Cauquenes & 16 & 8.6 \\
Curicó & 6 & 3.2 \\
\hline Total & 185 & 99.8 \\
\hline
\end{tabular}

Nota: Elaboración propia.

\section{Frecuencia de sesiones con el tutor en la universidad (tabla 5)}

Tabla 5

Frecuencia de sesiones con el tutor en la universidad

\begin{tabular}{lrr}
\hline Frecuencia de las sesiones & $\mathrm{f}$ & \multicolumn{1}{c}{$\%$} \\
\hline 1 vez por semana & 176 & 95.1 \\
Cada quince días & 4 & 2.1 \\
1 vez por mes & 2 & 1.0 \\
1 o 2 veces en el semestre & 3 & 1.6 \\
\hline Total & 185 & 99.8 \\
\hline
\end{tabular}

Nota: Elaboración propia.

Frecuencia de visitas del personal tutor al centro de práctica (tabla 6)

Tabla 6

Frecuencia de visitas del personal tutor al centro de práctica

\begin{tabular}{llr}
\hline Frecuencia de visitas del personal tutor al centro de práctica & \multicolumn{1}{c}{ f } & \multicolumn{1}{c}{$\%$} \\
\hline 1 vez por semana & 17 & 9.1 \\
Cada quince días & 20 & 10.8 \\
1 vez por mes & 48 & 25.9 \\
1 o 2 veces en el semestre & 18 & 9.7 \\
3 veces al semestre & 82 & 44.3 \\
\hline Total & 185 & 99.8 \\
\hline
\end{tabular}

Nota: Elaboración propia. 


\section{Estilo de trabajo con el personal tutor: Identificación de fortalezas y debilidades (tabla 7)}

Tabla 7

Estilo de trabajo con el personal tutor: Identificación de fortalezas y debilidades

\begin{tabular}{lcc}
\hline Estilo de trabajo con el personal tutor & $\mathrm{f}$ & $\%$ \\
\hline $\begin{array}{l}\text { "Como practicante escucho al profesor tutor cuando identifica fortalezas, debilidades y errores } \\
\text { y me señala lo que debo mejorar" }\end{array}$ & 126 & 68.1 \\
$\begin{array}{l}\text { "Como practicante señalo mis fortalezas, debilidades y errores, y el profesor tutor me ayuda en } \\
\text { el análisis" }\end{array}$ & 5931.9 \\
\hline Total & 185 & 100 \\
\hline
\end{tabular}

Nota: Elaboración propia.

Desde el punto de vista del trabajo con el personal tutor, el sujeto practicante, en su gran mayoría (68.1\%) reconoce un estilo de interacción donde es el personal tutor quien le señala los aspectos a mejorar, a partir de la identificación de fortalezas, debilidades y errores cometidos. En cambio, el 31.9\% concibe un estilo de trabajo centrado en la relación de ayuda del personal tutor para que sea cada estudiante quien analice sus fortalezas y debilidades.

\section{Estilo de trabajo con el personal tutor: Análisis de situaciones (tabla 8)}

Tabla 8

Estilo de trabajo con el personal tutor: Análisis de situaciones

\begin{tabular}{lcc}
\hline Estilo de trabajo con el personal tutor & $f$ & $\%$ \\
\hline $\begin{array}{l}\text { "El análisis de las acciones, decisiones o interpretaciones vividas por mí en la práctica está } \\
\text { centrado en cuán bien realizo las diversas tareas" }\end{array}$ & 67 & 36.2 \\
$\begin{array}{l}\text { "El análisis de las acciones, decisiones o interpretaciones vividas por mí en la práctica está } \\
\text { centrado en el impacto en el aprendizaje de los niños/adolescentes con los cuales trabajo" }\end{array}$ & 118 & 63.8 \\
\hline Total & 185 & 100 \\
\hline
\end{tabular}

Nota: Elaboración propia.

En relación con el análisis de situaciones en los espacios de tutoría, el 63.8\% alude a un ejercicio cuyo foco está centrado en el impacto que el trabajo tiene en el aprendizaje estudiantil, en tanto, el $36,2 \%$ centra el análisis en la gestión del sujeto practicante, en definitiva en la enseñanza y específicamente en la eficacia de la intervención. 
doi: http://dx.doi.org/10.15359/ree.20-2.17

URL: http://www.una.ac.cr/educare

CORREO: educare@una.cr

\section{Estilo de trabajo con el personal tutor: Propósito de la retroalimentación (tabla 9)}

Tabla 9

Estilo de trabajo con el personal tutor: Propósito de la retroalimentación

\begin{tabular}{lrc}
\hline Estilo de trabajo con el personal tutor : propósito de la retroalimentación & $\mathrm{f}$ & $\%$ \\
\hline La retroalimentación busca potenciar la toma de decisiones del sujeto practicante & 71 & 38.3 \\
La retroalimentación busca potenciar la correcta aplicación de conceptos y procedimientos & 114 & 61.6 \\
\hline Total & 185 & 99.9 \\
\hline
\end{tabular}

Nota: Elaboración propia.

En el contexto de la retroalimentación que da sentido al trabajo de tutoría, es posible constatarqueel $61.6 \%$ deestudiantes en práctica atribuye como propósito dela retroalimentación el potenciar la correcta aplicación de conceptos y procedimientos, declarando con ello una centralidad en la enseñanza. Por otro lado, el 38.3\% considera que el propósito está referido al fortalecimiento de la capacidad de toma de decisiones en el espacio del aula y, por lo tanto, más en la línea de centralidad en el aprendizaje del alumnado del sistema escolar.

\section{Orientaciones del personal tutor para apoyar el aprendizaje del estudiantado en práctica (tabla 10)}

Tabla 10

Orientaciones del personal tutor para apoyar el aprendizaje del estudiantado en práctica

\begin{tabular}{lrr}
\hline Orientaciones del personal tutor para apoyar el aprendizaje & $\mathrm{f}$ & $\%$ \\
\hline Clarificar qué hacer & 7.7 & 52 \\
Orientar cómo hacer & 118 & 63.7 \\
Evaluar, reflexionar, retroalimentar lo realizado & 2 & 1.0 \\
Analizar aspectos operativos y administrativos del centro de práctica & 6 & 3.2 \\
Apoyar la clarificación de la vocación y comprensión de la profesión docente & 185 & 99.7 \\
\hline Total & 2 & 2 \\
\hline
\end{tabular}

Nota: Elaboración propia. 
Consultados de las orientaciones recibidas por el personal tutor, el 63.7 alude a evaluar, reflexionar, y retroalimentar lo realizado en el aula; en tanto el $28.1 \%$ considera orientaciones referidas a cómo intervenir en la práctica y asegurar la adecuada gestión.

\section{Actividades desarrolladas en la tutoría para apoyar al sujeto practicante (tabla 11)}

Tabla 11

Actividades desarrolladas en la tutoría para apoyar al sujeto practicante

\begin{tabular}{lrc}
\hline Actividades desarrolladas en la tutoría & $\mathrm{f}$ & $\%$ \\
\hline Trabajo a partir de referentes teóricos didácticos & 13 & 7.0 \\
Trabajo con énfasis en las experiencias en el centro de práctica & 127 & 68.6 \\
Trabajo con énfasis en aprender a reflexionar & 45 & 24.3 \\
\hline Total & 185 & 99.9 \\
\hline
\end{tabular}

Nota: Elaboración propia.

Las orientaciones recibidas en la tutoría se traducen en diversas actividades desarrolladas en dicho espacio. Así, el 68,6\% alude a un trabajo centrado en las experiencias que se tienen en práctica; mientras que el $24.3 \%$ las vincula al aprender a reflexionar a partir de la experiencia. Por último, un 7\% refiere a un trabajo que se realiza a partir de referentes de carácter conceptual y didáctico.

\section{Discusión y conclusiones}

La formación inicial del profesorado supone una articulación teoría-práctica mediada por la reflexión, si lo que pretende es evitar el "desfase entre una formación académica más bien inservible y una práctica intuitiva que puede resolver los imprevistos y problemas circunstanciales pero nos da la impresión de un eterno comienzo" (Bélair, 2010, p. 105).

Ese planteamiento pone de manifiesto la importancia de una decisión clara respecto a la relación teoría-práctica en la formación del profesorado, consciente de sus implicancias en el tipo de docente que termina por configurar; y la necesidad de articular adecuadamente la triada conocimiento, contextos y actores, superando las lógicas dicotómicas y excluyentes, pues en caso contrario la teoría sería letra muerta y la práctica una acción rutinaria. 
doi: http://dx.doi.org/10.15359/ree.20-2.17

URL: http://www.una.ac.cr/educare

CORREO: educare@una.cr

Toda la evidencia teórica y que los datos empíricos de este estudio confirman, señala la importancia de "acelerar el ritmo de la alternancia entre la formación para el análisis conceptual y la formación para la intervención" (Carbonneau y Hétu 2010, p. 129).

El espacio que está llamado a facilitar la formación y una adecuada comprensión de la naturaleza del trabajo docente es el de la práctica.

Una práctica no reducida a un dominio de aplicación de teorías externas, sino más bien un lugar de constante producción de nuevas soluciones a los problemas con los cuales se ven enfrentados los grupos profesionales. De ahí la necesidad de no limitar la práctica al aprender a enseñar en el aula, pues ello equivale, en la mirada de Davini (1995), a "desestimar sus dimensiones sociocultural y ético política" (p.114), marginando el rol que juega la subjetividad y los propios marcos interpretativos del futuro profesorado, en la comprensión de los procesos de enseñanza-aprendizaje.

Coherente con esta relevancia, la institución ha decidido la implementación de un sistema de tutoría, según la cual el personal tutor está llamado a ofrecer el apoyo necesario en el proceso formativo del estudiantado de pedagogía. En ese proceso de acompañamiento emergen los aspectos más controvertidos del trabajo docente, más allá de las declaraciones planteadas por los proyectos formativos de las distintas carreras o las declaraciones de perfiles de egreso que están llamados a direccionar las distintas titulaciones. Un aspecto central que es develado en este espacio es el referido a la compleja relación teoría-práctica; y desde esta, las herramientas que finalmente dispone el sujeto practicante para enfrentar la complejidad del aula.

En el escenario de la relación teoría-práctica, es posible reconocer la existencia de estudiantes en distintos estadios de comprensión de esta relación, la cual podría explicarse por las interacciones que se desarrollan entre las características personales desde las cuales se leen y significa la naturaleza del trabajo docente y las vivencias rescatadas de su inserción en los contextos de aula. Se observa la presencia de un continum de estadios que va desde las representaciones según la cual teoría y práctica constituyen realidades opuestas, cuando se reconoce que: "Muchas ocasiones nos encontramos con situaciones que se escapan de los conceptos teóricos que se nos enseña en la educación superior, recurriendo a criterios, recursos personales e intuitivos o competencias desarrolladas a través del tiempo" $(\mathrm{H} 3)$; o "cuando estoy en la sala de clases y enfrento a mis estudiantes, lo hago apelando a mi sentido común o a mis recuerdos de profesores" (H7); pasando por aquellas representaciones centradas en la predominancia de una dimensión sobre la otra y que se traduce en dos vertientes.

La primera reflejada en la lógica según la cual la teoría es previa a la práctica, se encuentra presente en la idea según la cual "es fundamental tener los conocimientos teóricos para poder aplicarlos en la sala de clases. Si no tengo claro qué tengo que enseñar voy a tener problemas en el aula" (C5); o "Personalmente creo que un buen profesional debe primero adquirir el 
conocimiento necesario para luego ponerlo en práctica y no cometer errores irreparables, más aún, perfeccionarse día a día" (F1); y la segunda, según la cual la práctica es previa a toda teoría, en cuyo caso nos encontramos con relatos en la línea de "Es finalmente en la práctica donde el alumno logra modelar su perfil específico de docente como una mezcla de conocimiento teórico, personalidad y formación inicial y las necesidades que el propio contexto del establecimiento educativo le presenta como desafío profesional" (B1) y "reconocer la individualidad, ambiente, historicidad, elementos demográficos, etc. son elementos decisivos que se deben considerar antes de implementar una unidad de aprendizaje, y que uno los descubre en el terreno y a partir de estas necesidades emerge la preocupación por la teoría" (F5).

Finalmente, un grupo menor de estudiantes ha logrado articular una representación donde práctica y teoría se encuentran relacionadas y articuladas por la reflexión. Así lo refieren relatos del tipo "la teoría y la práctica están íntimamente ligadas. La práctica sirve para entender y confirmar la teoría. La teoría sirve para evitar improvisaciones y dirigirla en forma ordenada" (B4) o"si yo no puedo aprender del modo que tú enseñas, ¿me podrías enseñar de modo que yo aprenda?, es lo que he reflexionado en mi formación" (H8). Como lo reporta la investigación, las respuestas de quienes participaron en el estudio ponen de manifiesto la presencia de distintos paradigmas, lo cual determina no solo que las perspectivas de la relación teoría-práctica sean diferentes, sino también que los modos de acción difieran.

En los relatos emerge la complejidad del encuentro teoría-práctica reflejada en el conocido choque de realidad. De acuerdo con este, "todo transcurre como si, los conocimientos y las imágenes que sustentaban tanto las esperanzas de la profesión como de uno mismo en su práctica no se correspondieran con las situaciones concretas que, día a día, deben afrontarse sobre el terreno" (Baillauqués, 2010, p. 66). Enfrentado a este choque se realidad, el estudiantado no estaría evidenciando un desarrollo suficiente de las competencias que le permitan entender la complejidad y naturalidad de ese choque, dada la especificidad de la tarea docente, y las herramientas desde las cuales asumirlo de manera más integrada y no parcelada o normativa.

Al enfrentar este choque de realidad, el estudiantado muestra aún disparidad en el desarrollo de la relación compleja teoría-práctica; con una parte que ha logrado comprender la complejidad de la relación y la especificidad del trabajo docente; mientras que otros grupos aún siguen anclados a posiciones dicotómicas, según la cual teoría y práctica son realidades opuestas. La gran mayoría concibe la relación en términos de preponderancia de una respecto a otra, sea en la lógica de teoría-práctica o práctica-teoría. La implicancia de estas opciones y estadios es que, finalmente, los comportamientos pedagógicos y relacionales resultarán afectados por todo esto y pueden someterles, finalmente, a modelos de enseñanza considerados tradicionales, dado que enfrentan una realidad que no siempre se condice con sus representaciones de la naturaleza de la enseñanza. 
doi: http://dx.doi.org/10.15359/ree.20-2.17

URL: http://www.una.ac.cr/educare

CORREO: educare@una.cr

Estas representaciones de la relación teoría-práctica ponen de manifiesto ciertas lógicas de comprensión y actuación en el espacio educativo de los grupos profesionales en formación. En primer lugar, sus esquemas conceptuales son aún insuficientes para enfrentar la inserción a los contextos escolares reales y asumir la complejidad del aula; su percepción de la realidad es parcial y tendrían dificultades en el dominio de las situaciones que viven, razón por la cual generan representaciones de oposición teoría-práctica, o de predominancia de una sobre la otra, y son menos quienes logran avanzar a la idea de articulación, alcanzando así mayor conciencia de la verdadera especificidad de la tarea docente de aula.

En segundo lugar, al enfrentar los desafíos del aula, su formación parece bruscamente demandada, perdiendo control sobre las situaciones, pues nada o muy poco se comporta como estaba previsto; como debía ser o como esperaba que fuera. En ese sentido, emergen representaciones estereotipadas como aquellas que aluden a la ilusión del poder interventor de la teoría sobre la práctica, evidenciando básicamente rigidez para pensar y actuar. La mayoría de estudiantes muestra una percepción situacional aun reducida.

Por último, los grupos profesionales en formación tien den a la fragmentación de la realidad, resultándoles difícil comprender la especificidad del trabajo docente, pues se lo plantean en términos en general dicotómicos o excluyentes.

Desde la perspectiva de una parte de estudiantes: “La dicotomía se produce porque en la escuela se convive con múltiples variables que condicionan o potencian el quehacer del docente. Este proceso es dinámico, las clases no son iguales pese a que debemos lograr el mismo aprendizaje esperado, cada curso y nivel tiene sus propias complejidades. Así uno comienza a constatar que la teoría se aleja de la práctica y en ese sentido la reflexión se torna trascendental para aprender de lo que se está viviendo en el aula. No obstante, no son excluyentes van de la mano, por ejemplo, no podemos desenvolvernos adecuadamente en un contexto particular si se prescinde de uno de estos elementos, cuando trabajo en la práctica está implícita la teoría lo que ocurre es que no siempre nos damos cuenta" $(\mathrm{H} 2)$

En ese escenario, la tutoría enfrenta la disparidad de representaciones respecto al trabajo docente y la naturaleza de las relaciones teoría-práctica por parte del grupo profesional en formación. Además, la tutoría tampoco estaría logrando todas las condiciones necesarias para tensionar esas representaciones y favorecer aquellas más adecuadas a un trabajo en que el oficio de maestro o maestra, "por el hecho de abarcar un conjunto de tareas complejas y de exigir una serie de conocimientos ligados a la experiencia, debe ser enseñado en relación con esta complejidad y por medio de la organización personalizada de los aprendizajes respecto a este conjunto de tareas" (Bélair, 2010, p. 106). 
Lo anterior queda reflejado en una tutoría que, si bien ayuda al estudiantado en el análisis de sus fortalezas y debilidades, también cae en señalarle lo que "debe mejorar". Es decir, le permite el análisis de sus acciones, decisiones e interpretaciones vividas en relación con el impacto en el aprendizaje de la niñez-adolescencia con la cual trabaja; pero en ocasiones, el ejercicio se desplaza a cuán bien se realizan las tareas. Asumiendo la complejidad de la formación, la tutoría entregaría orientaciones al estudiantado en la línea de evaluar, reflexionar y retroalimentar lo realizado, a partir de un trabajo con énfasis en las experiencias en el centro de práctica.

Finalmente, el desafío a la formación inicial se plantea en términos de generar espacios y dispositivos que permitan develar las representaciones de los grupos profesionales en formación y, a partir de ahí, "familiarizar al aprendiz como unos juegos de reglas flexibles y cambiantes, en protegerlo frente a la ilusión de la explicación teórica todopoderosa, falaz e indispensable por el hecho de ser parcial y estereotipada" (Carbonneau y Hetú, 2010, pp. 122-123).

Para ello, resulta altamente recomendable plantear a la formación inicial: regular propositivamente el ritmo de alternancia entre formación para el análisis conceptual y para la actuación en contextos reales; multiplicar las situaciones de formación para las que el estudiantado de pedagogía tenga que aprender a elaborar sus respuestas propias; y ante todo, valorar el despertar a la conciencia de cada estudiante, acerca de sus propias representaciones sobre la docencia.

\section{Referencias}

Altet, M. (2010). La competencia del maestro profesional o la importancia de saber analizar las prácticas. En L. Paquay, M. Altet, E. Charliery P. Perrenoud (Coords.), La formación profesional del maestro. Estrategias y competencias (pp. 33-54). México: FCE.

Avalos, B. (2002). Profesores para Chile: Historia de un Proyecto. Santiago: Ministerio de Educación. Recuperado de http://biblioteca.uahurtado.cl/ujah/reduc/pdf/pdf/9161.pdf

Baillauquès, S. (2010). El trabajo de las representaciones en la formación de los maestros. En L. Paquay, M. Altet, É. Charlier y P. Perrenoud (Coords.), La formación profesional del maestro. Estrategias y competencias (pp. 55-87). México: FCE.

Bélair, L. (2010). Enseñar la complejidad del oficio de maestro. En L. Paquay, M. Altet, É. Charlier y P. Perrenoud (Coords.), La formación profesional del maestro. Estrategias y competencias (pp. 88-106). México: FCE.

Boggino, N. y Rosekrans, K. (2004). Investigación-acción: Reflexión crítica sobre la práctica educativa. Orientaciones prácticas y experiencias. Rosario: HomoSapiens. 
doi: http://dx.doi.org/10.15359/ree.20-2.17

URL: http://www.una.ac.cr/educare

CORREO: educare@una.cr

Carbonneau, M. y Hétu, J. (2010). La formación práctica de los maestros y el nacimiento de una inteligencia profesional. En Paquay, L., Altet, M., Charlier, E., y Perrenoud, P. (Coords.), La formación profesional del maestro. Estrategias y competencias (pp. 107-138). México: FCE.

Carr, W. y Kemmis, S. (1986). Teoría crítica de la enseñanza. La investigación acción en la formación del profesorado. Barcelona: Martínez Roca.

Correa, E., Cividini, M., Fuentealba (2013). Formación inicial e inserción profesional: Procesos clave para la profesionalización docente. En E. Corrrea, M. Cividini y M. Fuentealba (Eds.), Formación e inserción profesional: Desafíos y pistas de facilitación para la profesionalización docente (pp. 9-18). Santiago: OEI

Davini, M. C. (1995). Formación docente en cuestión: Política y pedagogía. Buenos Aires: Paidós.

Diker, G. y Terigi, F. (1997). La formación de maestros y profesores: Hoja de ruta. México: Paidós.

Flick, U. (2007). Introducción a la investigación cualitativa. Madrid: Morata.

Fuentealba, R. y Sánchez, G. (2013). Los tutores como posibilitadores del tercer espacio en la formación práctica, ¿utopía o realidad? En E. Correa, M. Cividini y M. Fuentealba, I. (Eds.), Formación e inserción profesional: Desafíos y pistas de facilitación para la profesionalización docente (pp. 65-89). Santiago: OEI

Fuentes, D., Vásquez, I. y Vergara, J. (2013). Formación inicial docente: Hacia una práctica profesional amigable. En A. Galaz, R. Fuentealba, J. Cornejo, A. Padilla (Eds.), Estrategias reflexivas en la formación de profesores y de formadores de profesores. ¿Qué desafíos se proyectan desde la formación basada en competencias (pp. 63-81) Santiago: LOM.

Hernández, R., Fernández, C., Baptista, P. (2010). Metodología de la investigación. México: McGraw-Hill.

Krause, M. (1995). La investigación cualitativa. Un campo de posibilidades y desafíos. Revista Temas de Educación, 7, 19-39. Recuperado de https://investiga-aprende-2.wikispaces. com/file/view/Inv-cualitat-Krause.pdf

Padilla, A. y Pedreros, A. (2013). Enseñando a enseñar: ¿Qué papel cabe a la reflexión en el desarrollo del pensamiento didáctico en la formación inicial de profesores. En A. Galaz, R. Fuentealba, J. Cornejo, A. Padilla (Eds.), Estrategias reflexivas en la formación de profesores y de formadores de profesores. ¿Qué desafíos se proyectan desde la formación basada en competencias (pp. 63-81) Santiago: LOM.

Paquay, L. y Wagner, M.-C. (2005). Formación continua y videoformación: Qué habilidades se deben priorizar. En Paquay, L., Altet, M., Charlier, E., y Perrenoud, P. (Coords.), La formación profesional del maestro. Estrategias y competencias (pp. 222-264). Madrid: FCE. 
Sánchez, G. (2014-15). Visión del trabajo docente desarrollada por estudiantes en proceso de inserción al aula. Santiago, Chile: Facultad de Ciencias de la Educación, Universidad Católica del Maule.

\section{(9) Cómo citar este artículo en APA:}

Sánchez-Sánchez, G. I. (Mayo-agosto, 2016). Relación teoría-práctica entre el campo de la formación inicial y el escenario del ejercicio profesional. Revista Electrónica Educare, 20(2), 1-25. doi: http:// dx.doi.org/10.15359/ree.20-2.17

Nota: Para citar este artículo en otros sistemas puede consultar el hipervínculo "Como citar el artículo" en la barra derecha de nuestro sitio web: http://www.revistas.una.ac.cr/index.php/EDUCARE/index 